\title{
Mandenkan
}

MANDENIKAN Bulletin semestriel d'études linguistiques mandé

49 | 2013

Le maninka du Niokolo

\section{Pronoms personnels, réfléchis et réciproques}

Section 11

\section{Denis Creissels}

\section{(2) OpenEdition}

Journals

Édition électronique

URL : https://journals.openedition.org/mandenkan/607

DOI : 10.4000/mandenkan.607

ISSN : 2104-371X

Éditeur

Llacan UMR 8135 CNRS/Inalco

\section{Édition imprimée}

Date de publication : 1 juin 2013

Pagination : 84-87

ISSN : 0752-5443

\section{Référence électronique}

Denis Creissels, «Pronoms personnels, réfléchis et réciproques », Mandenkan [En ligne], 49 | 2013, mis en ligne le 25 avril 2014, consulté le 16 janvier 2023. URL : http://journals.openedition.org/ mandenkan/607 ; DOI : https://doi.org/10.4000/mandenkan.607

Ce document a été généré automatiquement le 16 janvier 2023.

\section{(c) $)(1)(2)$}

Creative Commons - Attribution - Pas d'Utilisation Commerciale - Partage dans les Mêmes Conditions 4.0 International - CC BY-NC-SA 4.0

https://creativecommons.org/licenses/by-nc-sa/4.0/ 


\title{
Pronoms personnels, réfléchis et réciproques
}

\author{
Section 11
}

Denis Creissels

\subsection{Les pronoms personnels}

\subsubsection{Pronoms personnels emphatiques et non emphatiques}

1 Il y a deux séries de pronoms personnels qui ne présentent aucune différence de distribution. La série caractérisée par le marqueur -te' (dont le ton haut flottant n'est apparent que si la syllabe précédente ne comporte pas de ton haut) implique seulement une certaine emphase. La série emphatique n'est pas spécialisée dans une fonction discursive particulière, et se rencontre aussi bien en contexte de topicalisation qu'en contexte de focalisation.

2 Morphologiquement, la forme emphatique est marquée par un suffixe -te', et inclut en outre le marqueur de pluriel aux trois personnes du pluriel. La 2ème personne du pluriel a deux variantes aussi bien dans la série emphatique que dans la série nonemphatique, et l'une des deux variantes emphatiques a la particularité de ne pas être formée sur la forme non-emphatique du pronom de 2ème personne du pluriel, mais sur la forme non-emphatique de la 2ème personne du singulier. Aucune différence de sens ou de distribution n'a été relevée entre ces variantes de la 2ème personne du pluriel.

\begin{tabular}{|l|l|l|l|}
\hline & non-emphatique & emphatique \\
\hline $1 \mathrm{sg}$ & $y$ & $n t e^{\prime}$ \\
\hline $2 \mathrm{sg}$ & $i$ & ite' \\
\hline $3 \mathrm{sg}$ & Ca & áte \\
\hline
\end{tabular}




\begin{tabular}{|l|l|l|l|}
\hline & $1 \mathrm{pl}$ & $\dot{y}$ & ńte lu \\
\hline & $2 \mathrm{pl}$ & o álu & oté lu ité lu \\
\hline & $3 \mathrm{pl}$ & $\hat{i}$ & íte lu \\
\hline
\end{tabular}

3 On doit ajouter à cet inventaire un pronom inclusif aluín qui a la particularité de pouvoir apparaître seulement en combinaison avec le prédicatif du subjonctif (positif ou négatif) pour exprimer l'impératif inclusif (cf. section 4.2.4.3).

\subsubsection{Emplois spéciaux des pronoms personnels}

4 Comme de manière générale en mandingue, le pronom de 3ème personne du pluriel peut s'utiliser en référence, non seulement à une pluralité d'individus connus de l'énonciateur et identifiables par l'interlocuteur, mais aussi à un groupe indéterminé de personnes, et le pronom de 2ème personne du singulier peut s'utiliser en valeur générique. Dans l'emploi générique du pronom de 2ème personne du singulier, on remarque la possibilité qu'il reprenne un antécédent explicitant le domaine sur lequel porte la généralisation (à l'ex. (121) móxǒo 'l'être humain', à comprendre ici en valeur générique et non pas anaphorique : 'l'être humain en général').

\begin{tabular}{|c|c|c|c|c|c|c|c|c|c|}
\hline$(121)$ & \multicolumn{2}{|c|}{ Móyǒo } & $m e-i e$ & \multicolumn{2}{|l|}{$i$} & \multicolumn{2}{|c|}{ níi-kundán-o } & \multicolumn{2}{|l|}{ log, } \\
\hline & \multicolumn{2}{|c|}{ personne.D } & РОТР & \multicolumn{2}{|l|}{$2 S G$} & \multicolumn{2}{|c|}{ offrir-plat-D } & \multicolumn{2}{|l|}{ connaître } \\
\hline & \multicolumn{9}{|c|}{ 'Tu ne reconnais pas la portion de nourriture qui t'est destinée, } \\
\hline & \multicolumn{9}{|c|}{ litt. 'L'homme ne reconnait pas la portion de nourriture qui t'est destinée' } \\
\hline & bári & \multicolumn{2}{|l|}{$i$} & niii-kumáa, & $i$ & ye & wóo & log & née. \\
\hline & mais & \multicolumn{2}{|c|}{$2 S G$} & offrir-parole.D & $2 S G$ & ACPP & DEM & connaître & FOC \\
\hline & mais & & est & stinée, tu la r & con & & & & \\
\hline
\end{tabular}

\subsection{Le pronom réfléchi}

5 Le maninka du Niokolo a un pronom réfléchi avec les deux formes $y$ et $i$. La forme $\eta$ s'utilise si le sujet est de première personne (singulier ou pluriel), et la forme $i$ si le sujet est de 2ème ou 3ème personne (singulier ou pluriel), ce qui donne par exemple le paradigme suivant avec le verbe kúu 'laver' :

\begin{tabular}{|l|l|l|}
\hline & y yá y kúu & 'je me suis lavé(e)’ \\
\hline i yé i kúu & 'tu t'es lavé(e)’ \\
\hline
\end{tabular}




\begin{tabular}{|l|l|l|}
\hline & á ye i kúu & 'il/elle s'est lavé(e)' \\
\hline & ý ya y kúu & 'nous nous sommes lavé(e)s \\
\hline & o yé i kúu & 'vous vous êtes lavé(e)s' \\
\hline & álu yé i kúu & \\
\hline & i ye i kúu & 'ils/elles se sont lavé(e)s \\
\hline
\end{tabular}

6 Le pronom réfléchi apparaît presqu'exclusivement en position d'objet, et même dans le rôle d'objet sa productivité est limitée. Ce sont les pronoms intensifs (cf. 11.3) qui constituent en maninka du Niokolo la façon productive d'exprimer la réflexivité.

7 Le seul emploi possible du pronom réfléchi en dehors de la position d'objet est dans la formation des pronoms intensifs. En effet, le pronom intensif de troisième personne est en principe $a$ emphatique est co-référent d'un sujet de troisième personne (cf. 11.3)

8 Par ailleurs, avec certains verbes, le pronom réfléchi peut figurer en position d'objet sans qu'il y ait pour autant réflexivisation. Deux cas sont à distinguer. Il y a d'abord des verbes comme bóri 'courir, s'enfuir' ou dón 'danser', qui s'utilisent avec le pronom réfléchi pour encoder un événement à un seul participant qui ne peut pas s'interpréter comme la variante réfléchie d'un événement à deux participants - ex. (122).

\begin{tabular}{|l|l|l|l|l|l|l|l|l|}
\hline$(122)$ & a. & Á & ye & $i$ & bóri & dulaa & ján-o & to. \\
\hline & & 3SG & ACPP & REFL & courir & endroit & être_éloigné-D & LOC \\
\hline & \multicolumn{6}{|c|}{ 'Il s'est enfui au loin.' } \\
\hline
\end{tabular}

\begin{tabular}{|l|l|l|l|l|l|l|l|l|}
\hline & b. & Sikăa & lu & bé & i & dón-na & bantabáa & to. \\
\hline & & masque & PL & COPLOC & REFL & danser-INF & place_publique.D & LOC \\
\hline & \multicolumn{6}{|c|}{ 'Les masques dansent sur la place publique.' } \\
\hline
\end{tabular}

9 Il y a ensuite des verbes comme ñaalatómboy 'choisir', qui s'utilisent en construction réfléchie avec un complément oblique encodant un participant qui pourrait aussi apparaître comme l'objet d'une construction transitive - ex. (123). Dans ce cas, le pronom réfléchi assume la fonction d'un opérateur de valence de type antipassif.

\begin{tabular}{|l|l|l|l|}
\hline$(123)$ & a. & Mang-óo & ñaalatómboy! \\
\hline & & mangue-D & choisir \\
\hline & & 'Choisis une mangue!' \\
\hline
\end{tabular}




\begin{tabular}{|l|l|l|l|l|l|}
\hline & b. & I & ñaalatómbǒn & mang-óo & to! \\
\hline & & REFL & choisir & mangue-D & LOC \\
\hline & \multicolumn{4}{|l}{ même sens que (a) } \\
\hline
\end{tabular}

Dans cet emploi du pronom réfléchi, l'objet dont la place est prise par le pronom réfléchi peut être converti en oblique, comme en (123), mais il peut aussi rester non spécifié pour exprimer une valeur de généralisation, notamment avec je 'voir' et mo íyi 'entendre'. A l'ex. (124), mi yee est la réalisation de mer.e i.

\begin{tabular}{|c|c|c|c|c|}
\hline (124) & a. & Fionkinte & mi yee & je. \\
\hline & & aveugle.D & POTN.REFL & voir \\
\hline & & \multicolumn{3}{|c|}{ 'L'aveugle n'y voit pas. } \\
\hline
\end{tabular}

\begin{tabular}{|l|l|l|l|l|}
\hline & b. & Tulukuti-yy-o & mi-yee & mo $y$ yi. \\
\hline & & sourd-D & POTN.REFL & entendre \\
\hline & & \multicolumn{2}{|l}{ 'Le sourd n'entend pas. } \\
\hline
\end{tabular}

\subsection{Les pronoms intensifs}

11 Les pronoms intensifs sont formés en combinant les pronoms personnels avec la particule intensive fắ(o). Ils fournissent notamment la façon productive d'encoder la réflexivité. L'exemple (125) permet de constater qu'en fonction de réfléchi, le pronom intensif de 3ème personne du singulier peut de manière équivalente être $f a$ fou i făy(o) (où $i$ est le pronom réfléchi, qui en dehors de cet emploi particulier apparaît exclusivement en position d'objet).

\begin{tabular}{|l|l|l|l|l|l|l|l|l|}
\hline$(125)$ & a. & I & făjo & yé & $i$ & la & kúrt-óo & fara. \\
\hline & & 2SG & INT & ACPP & 2SG & GEN & pantalon-D & déchirer \\
\hline & \multicolumn{6}{|c|}{ 'Tu as déchiré ton pantalon toi-même.' } \\
\hline
\end{tabular}

\begin{tabular}{|l|l|l|l|l|l|l|l|}
\hline & b. & A & běe & ye & i & fằ & táa! \\
\hline & & $3 S G$ & tous & ACPP & $2 S^{1}$ & INT & prendre \\
\hline & \multicolumn{6}{|c|}{} \\
\hline
\end{tabular}




\begin{tabular}{|c|c|c|c|c|c|c|c|c|c|c|c|}
\hline c. & $A$ & ye & $a$ & fäyo & bárama. & $=$ & $A$ & ye & $i$ & fäno & bárama. \\
\hline & $3 S G$ & ACPP & $3 S G$ & INT & blesser & & $3 S G$ & ACPP & REFL & INT & blesser \\
\hline & 'Il & & & & e.' & & & & & & \\
\hline
\end{tabular}

\subsection{Le pronom réciproque}

12 La réciprocité est encodée en maninka du Niokolo au moyen du pronom réciproque ñoyo' occupant une position syntaxique autre que celle de sujet. A l'ex. (126), la phrase (c) illustre un contexte dans lequel le ton haut flottant final de ñoyo' laisse une trace de sa présence.

\begin{tabular}{|c|c|c|c|c|c|c|c|}
\hline (126) & a. & Náa & $f_{0}$ & $\dot{y}$ & ga & ñoyó & je! \\
\hline & & venir & jusqu’à & $1 \mathrm{PL}$ & SUBJP & RECIP & voir \\
\hline & & \multicolumn{6}{|c|}{ 'Viens pour que nous nous voyions!' } \\
\hline
\end{tabular}

\begin{tabular}{|c|c|c|c|c|}
\hline b. & 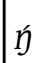 & be & ñoyo & déemaa-kaín. \\
\hline & 1PL & COPL & RECIP & aider-PROG \\
\hline \multicolumn{5}{|c|}{ 'Nous nous entraidons.' } \\
\hline
\end{tabular}

\begin{tabular}{|l|l|l|l|l|l|l|l|}
\hline & c. & I & ye & hake & tu & noyo- & yen. \\
\hline & & 3PL & ACPP & faute.D & laisser & RECIP & BEN \\
\hline & \multicolumn{6}{|c|}{ 'Ils se sont mutuellement pardonné.' } \\
\hline
\end{tabular}

\section{NOTES}

1. D'après le sens, on peut être tenté de reconnaître là le pronom réfléchi. Toutefois, compte tenu de l'aptitude du pronom de 2ème personne à reprendre des antécédents génériques, on peut penser qu'il s'agit plutôt du pronom de 2ème personne. 


\section{AUTEUR}

DENIS CREISSELS

Université de Lyon

Denis.Creissels@univ-lyon2.fr 